\title{
Extended mind and cognitive enhancement: moral aspects of cognitive artifacts
}

\author{
Richard Heersmink ${ }^{1}$
}

Published online: 30 November 2015

(C) Springer Science+Business Media Dordrecht 2015

\begin{abstract}
This article connects philosophical debates about cognitive enhancement and situated cognition. It does so by focusing on moral aspects of enhancing our cognitive abilities with the aid of external artifacts. Such artifacts have important moral dimensions that are addressed neither by the cognitive enhancement debate nor situated cognition theory. In order to fill this gap in the literature, three moral aspects of cognitive artifacts are singled out: their consequences for brains, cognition, and culture; their moral status; and their relation to personal identity.
\end{abstract}

Keywords Cognitive enhancement $\cdot$ Cognitive artifacts $\cdot$ Neuroethics $\cdot$ Extended mind . Distributed cognition $\cdot$ Material culture $\cdot$ Cognitive scaffolding

\section{Introduction}

Human beings enhance their cognitive abilities in a variety of ways, but most obviously with psychopharmaceuticals and external artifacts. The former are extensively debated by ethicists, whereas the latter are largely neglected by ethicists. In the major handbooks on neuroethics and cognitive enhancement (e.g., Savulescu and Bostrom 2009; Illes and Sahakian 2011; Hildt and Franke 2013; Clausen and Levy 2015) there is little, if any, talk of moral aspects of enhancing our cognitive abilities with external artifacts. Examples of such "cognitive artifacts" include navigation systems, diaries, diagrams, calculators, scale-models, timetables, textbooks, and computing devices (Heersmink 2014). Surprisingly, such artifacts and their moral aspects have not been addressed in the cognitive enhancement debate. Situated cognition theory, in contrast, has extensively studied cognitive artifacts (Norman 1993; Kirsh and Maglio 1994; Hutchins

Richard Heersmink

richard.heersmink@gmail.com

1 Department of Philosophy, Macquarie University, Sydney, Australia 
1995; Clark and Chalmers 1998), but also has not addressed their moral aspects in any substantial way. ${ }^{1}$ Therefore, as a contribution to a special issue on "Critiquing technologies of the mind: Enhancement, alteration, and anthropotechnology", the goal of this article is to start conceptualizing moral aspects of cognitive artifacts by drawing on and connecting the neuroethics of cognitive enhancement and situated cognition theory. It thereby fills a gap in the philosophical literature on artifacts and opens a dialogue between these fields.

The article begins by outlining the cognitive enhancement debate with a focus on conceptual issues related to "cognition" and "enhancement". Next, it briefly introduces situated cognition theory, suggesting that human cognition is scaffolded by and sometimes integrated with technological and social structures. Having set the stage, it then brings these debates together by presenting a number of moral issues pertaining to cognitive artifacts, including some of their consequences for brains, cognition, and culture; their moral status; and their relation to personal identity.

\section{What is cognitive enhancement?}

In the last two decades or so, a philosophical and ethical debate emerged about the enhancement of our cognitive abilities beyond medical or therapeutic purposes. In this debate, a number of cognitive enhancement methods and technologies are described and then evaluated in terms of their (potential) moral, anthropological, and societal consequences. In an overview paper, Martha Farah et al. explain that "In contrast to the other neurotechnologies mentioned earlier, whose potential use for enhancement is still hypothetical, pharmacological enhancement has already begun" (2004, p. 421). For this reason, the focus in the current debate is on moral aspects of psychopharmaceuticals (Turner and Sahakian 2006; Lynch et al. 2011). Emerging methods and technologies for cognitive enhancement like transcranial magnetic stimulation, brain-computer interfaces, brain implants, and genetic engineering, therefore, receive much less attention, but for an overview see (Bostrom and Sandberg 2009).

\subsection{Conceptual issues}

Two prominent conceptual issues in the debate are definitions and characterizations of "cognition" and "enhancement". It is difficult to find explicit definitions of cognition in the literature on enhancement. A notable exception are Nick Bostrom \& Anders Sandberg who write: "Cognition can be defined as the processes an organism uses to organize information". This includes acquiring information (perception), selecting (attention), representing (understanding), and retaining (memory) information, and using it to guide behaviour (reasoning and coordination of motor outputs) (2009, p. 312). Whilst their definition itself is rather broad (i.e., processes of organizing information), it remains silent on human embodiment and our interactions with the wider social-technological environment. An important aspect of human brains is that they are embodied and embedded (Haugeland 1998); if relevant features of our embodiment or environment are changed, then our cognitive abilities change as well. These aspects are

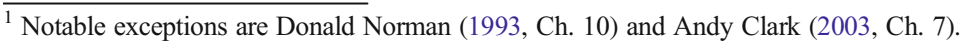


addressed neither by Bostrom \& Sandberg nor the cognitive enhancement debate more generally.

What cognition exactly is and how it is embodied and embedded is thus usually not explained. Theorists in the debate often give examples of specific cognitive abilities such as memory, attention, reasoning, and so on, to indicate what is enhanced. A clear example of this approach is put forward by the British Medical Association. They write: "The main aspects of cognition that we are interested in for the purpose of this paper are: learning, memory and information retrieval; concentration and attention; speed of processing; visuo-spatial ability; and executive functions including planning, and the ability to carry out abstract reasoning" (2007, p. 5).

One can hardly blame theorists in the enhancement debate for not clearly defining human cognition. In the philosophy of cognitive science, it has also proven difficult to give a clear definition of cognition (Buckner 2015). Historically, there have been many approaches to cognition. Behaviorism defines it as overt behaviour caused by environmental stimuli, without any reference to mental or psychological states. Cognitivism defines it as rule-based manipulations of symbols, which takes place in the brain and are shielded of from the wider external world. Cognitivism has been the dominant paradigm since the outset of the cognitive revolution in the $1950 \mathrm{~s}$, but has recently been challenged by situated approaches, including embodied, enactive, embedded, and extended approaches. These argue that human embodiment and the way we interact with the social-technological environment are essential for understanding human thought. So, "there is no standardized working definition of cognition on which neuroethicists could draw" (Metzinger and Hildt 2011, p. 245). One might ask whether this is a potential problem for the cognitive enhancement debate. No, it suffices, I think, to give exemplars of abilities that are generally accepted by the cognitive science community, including philosophers, as being cognitive. What is more important is to have a full understanding of the transformative effects of enhancement technologies on specific cognitive abilities such that ethicists can evaluate these effects, rather than to have a broad definition of cognition.

How might cognition be enhanced? Because human cognition is a richly multidimensional phenomenon, there are many methods, technologies, and strategies to enhance it. Education, mental training, textbooks, healthy diets, shopping-lists, goodquality sleep, calculators, caffeine, notebooks, mnemonics, Modafinil, maps, methods of loci, and computing devices, in one way or another, enhance our cognitive abilities. When cognition is enhanced, there is typically an increase in information-processing speed or capacity. Cognitive tasks are then performed faster, with less error, or made possible in the first place. On the basis of what does one distinguish between different kinds of cognitive enhancements? Depending on one's goal, there are various aspects one might focus on: (1) the cognitive ability that is enhanced (e.g., memory, attention, or reasoning); (2) the method or technology that enhances a cognitive ability (e.g., education, psychopharmaceuticals, or computers); or (3) the level at which the method or technology influences cognitive functioning (molecular/cellular (e.g., Modafinil) or conscious thought (e.g., education, mnemonics, or artifacts)). The later distinction is particularly relevant for this paper. Psychopharmaceuticals and artifacts both enhance cognition, but do so in very different ways: either by improving the molecularneurological basis for cognitive processing or by providing external information helpful for completing cognitive tasks. 
An often discussed distinction is one between treatment and enhancement. A child with ADHD taking Ritalin to improve focus and attention is seen as treatment, whereas a student taking Ritalin before an exam for the same reasons is seen as enhancement. Roughly, the idea is that treatment is considered as the goal of medicine and morally desirable, whereas enhancement is outside the scope of medicine and morally undesirable or at least seen as suspicious. The intuition is that a healthy person whose capabilities fall in the normal range does not need to be enhanced. Notions underlying this distinction are those of disease, health, and normal functioning (Metzinger and Hildt 2011). These notions are contingent on cultural and historical context and are thus difficult to define clearly. The paradigm cases of therapy and enhancement are clear, but the line between the two is rather fuzzy.

\subsection{Moral values}

Whilst the treatment/enhancement distinction is fuzzy, many moral considerations regarding cognitive enhancements are nonetheless based on this distinction. Ethicists have argued that various moral values are at stake when cognition is enhanced beyond medical or therapeutic purposes. Using psychopharmaceuticals has certain risks such as personality changes and emotional instability, which are often seen as undesirable by the user and family and friends (Hansson 2005). Furthermore, the long-term effects on the brain are unknown. It may turn out that Adderall, Ritalin, and Modafinil have effects on brain and cognition that are detrimental in the long run.

If cognitive enhancements become widespread, there might be situations in which people feel (are) pressured to enhance their cognitive abilities. Pilots, doctors, and other professionals with a high responsibility towards other people's safety or health might benefit from cognitive enhancers (Santoni de Sio et al. 2014). It might make flights and medical procedures safer and more efficient, due to a better memory and attention span. Employers and organizations will see the advantages of someone who has better memory, attention, or works more efficiently and will prefer someone who is cognitively enhanced (Farah et al. 2004). This may infringe the autonomy of doctors and pilots, but enhance safety of patients and passengers.

Some have argued that achieved skills have a higher worth than those which are bought. So if cognitive abilities are bought with a pill or some other enhancement, it would reduce their value and make them less admirable. It would also make those newly gained abilities inauthentic (Bostrom and Sandberg 2009). Access to shortcuts to better cognitive functioning would then become the determining factor of success and failure. Virtues like dedication, persistence, and willingness to work hard for one's goals might disappear if success requires only money (Turner and Sahakian 2006). If cognitive success is gained with buying enhancements, which are not available for everyone, it can be seen as a form of cheating. For example, healthy students taking Ritalin to enhance their focus during exams have an unfair benefit over those that did not take it.

Currently, cognitive enhancers for healthy people are not paid for by medical insurance. This means that only people who have access to enhancing technologies, are willing to take certain risks, and can afford them, will be able to enhance their cognition. It has been argued that this may increase the already large gap between certain social-economic groups, which is considered as unjust and unfair (BMA 2007). 
Thus, according to some theorists in the debate, moral values like safety, freedom, authenticity, equality, fairness, and others, may be compromised when cognition is enhanced beyond medical purposes.

\subsection{Psychopharmaceuticals versus external artifacts}

Compared to psychopharmaceuticals and emerging neurotechnologies such as braincomputer interfaces, external artifacts are more widespread, have a stronger and more obvious enhancing effect on cognition, enhance a broader spectrum of cognitive abilities, and have done so for a much longer period of time. For these reasons, it is surprising that the enhancement debate has neglected cognitive artifacts. An exception are again Bostrom \& Sandberg. "Education and training, as well as the use of external information processing devices, may be labelled as 'conventional' means of enhancing cognition" (2009, p. 312). Thus they recognize that external devices enhance cognition, but label them as conventional, which more or less implies that they are seen as morally and culturally accepted. This may be one of the reasons why artifacts are not sufficiently addressed by the cognitive enhancement debate. However, the attitude towards external artifacts as conventional, which seems to be fairly representative for the debate, runs the risk of narrowing the scope and ambition of the enhancement debate and neuroethics more generally. In order to start addressing this problem, I now turn to a view on cognition that puts external artifacts and material culture central.

\section{Situated cognition and cognitive scaffolding}

Situated cognition theory is a set of approaches to human cognition that underlines the importance of our embodied interactions with the social-technological environment for better understanding thought ${ }^{2}$ (Hutchins 1995; Clark 2003; Rowlands 2010). In an early statement of situated cognition, Donald Norman (1993) points out that the human mind is limited in capacity. The brain is a powerful information-processor, he says, but there are limits to its capacity to remember, calculate, navigate, plan, and learn. To overcome these limits, we create and use artifacts that scaffold us in performing cognitive tasks. We use diaries to help us remember our appointments, pen and paper to calculate, maps to navigate, diagrams to make inferences, timetables to plan, and textbooks to learn (Sternberg and Preiss 2005). Such cognitive artifacts complement (Sutton 2010) the information-storage and processing abilities of our embodied brains by proving task-relevant information.

Information held in conscious thought is often fleeting and so one advantage of externalizing thought is that it becomes fixed. Sometimes this information remains fixed, as in a textbook, but other times it is modified during a task, for example when making a calculation with pen and paper or writing an academic paper. So another advantage is that this allows us to perform operations on the externalized information that are very hard, if not impossible, to perform in the brain. Also, it is important to note

\footnotetext{
${ }^{2}$ This view is sometimes also referred to as $4 \mathrm{E}$ cognition, putting emphasis on the embodied, embedded, enactive, and extended nature of cognition. See Haugeland (1998), Kiverstein and Clark's (2009) special issue of Topoi, Menary's (2010a) special issue of this journal, or Ward and Stapleton (2012).
} 
that offloading information is not a recent phenomenon associated with the computer age and information society. We have offloaded information-storage functions to external media for thousands of years (Donald 1991), at least since the invention of written language captured in clay tablets, but probably even deeper into our evolutionary past. Minds and technologies have co-evolved at least since the dawn of language and so current "technologies of human cognitive enhancement are just one more step along this ancient human path" (Clark 2007, p. 278).

How exactly do artifacts enhance cognition? Following Norman (1991), a distinction can be made between the "personal view" and "system view" of cognitive artifacts. It might seem that artifacts enhance cognition by allowing us to accomplish cognitive tasks faster and with less error, but what they really do, Norman says, is transform one cognitive task into another. When pilots use a checklist rather than their biological memory, they make less mistakes. According to the system view, pilot-plus-checklist is an enhanced memory system because it is more reliable. However, according to the personal view, which is the subjective point of view of the artifact-user, the task is transformed from retrieving items from biological memory into retrieving items from the list. On this view, the list is not an enhancer because it does not improve biological memory, but merely transforms a task. Only when we take a systems perspective, can we speak of enhanced cognitive abilities.

Moreover, we do not just use artifacts to help us think, we also quite often rely on other people to complete our cognitive tasks. Psychologist Daniel Wegner (1995) describes how dyads and social groups develop transactive memory systems, which can be defined as a cognitive system shared by people in close relationships developed for encoding, storing, and retrieving information (see also Sutton et al. 2010). Celia Harris et al. (2010) describe an illustrative example of how transactive memory works. In their real-world example, a long married couple tries to recall the name of the show they saw on their honeymoon more than 40 years ago. Neither of them knows the name of the show, but by giving each other cues, they jointly construct the answer.

F: And we went to two shows, can you remember what they were called?

M: We did. One was a musical, or were they both? I don't ... no ... one ...

F: John Hanson was in it.

M: Desert Song.

F: Desert Song, that's it, I couldn't remember what it was called, but yes, I knew John Hanson was in it.

M: Yes

The above examples show that humans are creatures whose embodied brains are situated in a technological and social ecology of cognitive scaffolding. Such scaffolding enhances human cognition by providing information that is relevant for performing a cognitive task or by allowing us to manipulate and process the external information in a way that is difficult to do in our brain. Thus without artifacts and other people we 
would not have the same cognitive abilities. In some cases, agent and scaffolding are coupled in such a way that they can best be seen as a single cognitive system. When that happens, the cognitive system is extended or distributed (Hutchins 1995; Clark and Chalmers 1998; Menary 2010b; Rowlands 2010; Sutton et al. 2010). This occurs with both social and artifactual scaffolding, but given the goal of this paper I will focus on the latter.

To illustrate the notion of extended and distributed cognitive systems, consider Otto and his notebook (Clark and Chalmers 1998). Otto has Alzheimer's disease and thus battles with a decline in his ability to encode, store, and retrieve information. To compensate for his poor memory, he uses a notebook in which he writes important information such as names, addresses, phone numbers, things to do, observations, ideas, etc. The notebook is an essential tool for Otto. He deeply relies on it and always carries it with him so that the information is there when he needs it. Due to his poor memory, the notebook has become a substitute for his biological memory, playing the same causal roles as information stored in his biological memory. Clark \& Chalmers argue that what is central for external information to be constitutive of a cognitive state or process is a high degree of trust, reliance, and accessibility, and we must have endorsed it at some point in the past. These conditions are often referred to as "trust and glue". 3

Further developing the notion of a systems perspective, one may ask when people and artifacts are integrated into a unified cognitive system. This, I suggest, is a matter of degree and is best seen as a multidimensional phenomenon in which integration varies along a number of dimensions (Heersmink 2015). These dimensions include the kind and intensity of information flow between agent and scaffold, the accessibility of the scaffold, the durability of the coupling between agent and scaffold, the amount of trust a user puts into the information the scaffold provides, the degree of transparency-in-use, the ease with which the information can be interpreted, the amount of personalization, and the amount of cognitive transformation. The way we interact with external information may vary along all these dimensions and the higher a situated system ranks on these dimensions, the denser the integration between agent and scaffold.

Generally, if the integration is dense, the artifact can be seen as part of an extended cognitive system, whereas if it is shallow, the artifact can be seen as part of an embedded cognitive system. So there is an ontological difference between embedded and extended cognitive systems regarding the location of the physical substrate that realizes cognition. Extended and distributed views claim that scaffolding is, under certain conditions, constitutive of a cognitive state or process. Embedded views, in contrast, argue that artifacts and other environmental scaffolding have important roles in human cognitive functioning but are never part of cognition (e.g., Rupert 2004). I will come back to this distinction below in section 4.2, where I theorize about the moral status of cognitive artifacts in relation to their degree of integration.

This concise outline of situated cognition theory has shown that artifacts (and other people) have important cognition-enhancing functions. It has also shown that moral dimensions of such artifacts are not a prominent topic. To conceptualize situated

\footnotetext{
${ }^{3}$ This paper is not the place for an argument about the conditions of cognitive extension, but for discussion see (Sutton 2006; Menary 2010b; Sterelny 2010).
} 
cognitive system, theorists in this field typically focus on metaphysical, epistemological, and methodological notions, but rarely on moral ones.

\section{Moral aspects of cognitive artifacts}

Having briefly outlined the cognitive enhancement debate and situated cognition theory, I now aim to bring these debates together by presenting a general outline of moral issues related to cognitive artifacts. Some of the same moral values outlined above in section 2.2 such as authenticity, equal access, and fairness may be at stake when using cognitive artifacts. For instance, like taking Ritalin during an exam, using a smartphone with Internet access during an exam is seen as cheating and the results obtained as inauthentic (compare Wheeler 2011). Depending on various socialeconomic and cultural factors, we do not all have equal access to information, resulting in information haves and have-nots. So, like with most technologies, there is not an equal or fair distribution of cognitive technologies (see also Norman 1993, p. 202). Some traditional moral issues in the cognitive enhancement debate thus also apply to cognitive artifacts, which are important topics in need of further development. In this section, however, I focus on the consequences of cognitive artifacts for brains, cognition, and culture; the moral status of cognitive artifacts; and their relation to personal identity. These topics are central and distinctive to cognitive artifacts and have not received much attention. The goal of this section is not so much to argue in favor of a particular moral position, but to start addressing moral issues pertaining to cognitive artifacts.

\subsection{Consequences for brains, cognition, and culture}

In order to start an analysis of the consequences of cognitive artifacts, consider the following quote of Clark:

"It is our basic, biologically grounded nature (or so I have suggested) to be open to a wide variety of forms of technologically mediated enhancements (...) not all change is for the better, and hybridization (however naturally it may come to us) is neutral rather than an intrinsic good. Uncritical talk of human "enhancement" thus threatens to beg philosophically, culturally, and politically, important questions" (2007, p. 278, italics added).

Clark here suggests that it is in our biological nature to be open to enhance ourselves with technology. He claims that hybridization (i.e., our tendency to integrate tools and artifacts into our bodily, perceptual and cognitive systems) is not intrinsically good but neutral. He further claims that conceptualizing all enhancement as positive threatens to beg the question. We need not assume that all forms of hybridization are enhancements. This statement connects to the theme of this special issue. One of the potential topics of this special issue is not to use the term "enhancement" but "anthropotechnology", because enhancement is a value-laden term with a positive connotation, whereas anthropotechnology is descriptive (see also Meacham 2015). Clark is clearly aware of this issue, but he suggests that human-technology hybridization is neutral, echoing 
an instrumentalist view of technology. Let me explain why we should be careful with the term "neutral" in relation to technology-use or hybridization.

\subsubsection{Technological instrumentalism}

Technological instrumentalism is a view claiming that artifacts are mere value-neutral means to human ends (e.g., Pitt 2014). Technological artifacts merely connect a pregiven intention with a desired effect without shaping or influencing either. An often heard phrase in relation to instrumentalism is: "technology is not good or bad in itself, it is how you use it that determines its value". So, on this view, only the intentions of the user determine the value of technology. Very few philosophers of technology defend instrumentalism, because technologies do shape intentions and effects. They are furthermore not mere value-neutral instruments, but some artifacts are value-laden, which means they have moral values embedded in their structure. Also, whilst instrumentalism does not explicitly deny that artifacts can have unintended moral and societal consequences, it does not conceptualize these consequences sufficiently.

There are at least three ways to understand the non-neutrality of technology. Some argue that technological artifacts embody moral or political values. Bruno Latour (1994) argues that speed bumps force drivers to slow down, in that way embodying the value of traffic safety. Others, such as phenomenologist Don Ihde, argue that "technologies transform experience, however subtle, and that is one root of their nonneutrality" (1990, p. 49). Cars, telescopes, microscopes, walking canes, and hammers, for example, mediate and transform our experiences and perceptions of the world. Yet others argue that technology is not a mere means to an end, because artifacts have unintended consequences which are beyond our control. These scholars acknowledge that artifacts are used to achieve an end, but by doing so they also do unintended things. Cars are used for transportation, but they also emit carbon dioxide and contribute to global warming: an effect intended neither by the designers nor the users.

An instrumentalist view on cognitive artifacts would see them as mere neutral instruments used for completing our cognitive tasks. For instance, I may have an intention to drive from Sydney to Melbourne, but do not know how to get there, in which case I typically use my navigation system. However, navigation systems do much more than merely aiding us in navigating. My own navigation system beeps when I drive too fast. Whilst I can try to ignore the beeping, its function is nonetheless to make me aware that I am speeding and should slow down, in that way (like speed bumps) embodying traffic safety.

Navigation systems transform our experiences and perceptions of the world. They show points of interest like gas stations, car parks, and landmarks that are outside the scope of our direct perception, in this way allowing us to "see" things we could otherwise not have seen. Thus, phenomenologically, they transform our sense of the environment.

Navigation systems have unintended consequences: their users run the risk of becoming less skillful in navigating when they do not have access to their device. By relying too often and too much on such systems, their users do not (learn to) memorize routes, or to develop navigation skills by relying on orientation points. What they have learned is to incorporate the functionality of such devices into their navigational 
practices, which, in some cases at least, has a negative impact on the accuracy and level of detail of their internal cognitive maps (e.g., Burnett and Lee 2005).

So, whilst navigation systems are indeed instruments for realizing one's intentions and goals, they also embody moral values like safety, transform the experience of our environment, and have unintended consequences on our onboard cognitive capabilities. For these reasons, navigation systems and other cognitive artifacts are not mere neutral instruments, but morally significant objects.

\subsubsection{Cognitive transformation}

Now, Clark is no instrumentalist and is well-aware that cognitive technologies are not mere neutral instruments, but morally significant objects. Clark (2003, Ch. 7) points out a number of moral and societal issues related to cognitive technology such as unequal distribution of and access to cognitive technology; unauthorized access to personal information by individuals, corporations, and governments; information overload; and alienation. His attempt to address moral and societal issues is admirable, but one thing he does not address is how such technologies might transform our brains and cognition in certain ways. During our lifetime, we interact with cognitive artifacts from a very young age. Abacuses, textbooks, blackboards, tablets, and other computer devices populate classrooms and shape our brains and cognition in powerful ways. They do so most clearly in childhood and adolescence, but continue to do so throughout our lives. Through interacting with such artifacts, we internalize external representational systems such as language, number systems, and diagrams, thereby learning to think in terms of those systems (Menary 2010b). This hardly seems problematic from a moral perspective as the cognitive capabilities that result from these transformations largely define our human identity and have caused substantial progress for humans and society at large.

However, some theorists have argued that an overreliance on external information may cause a diminishing of some of our onboard cognitive capabilities and transform our brain and cognition in perhaps undesirable ways. Consistently outsourcing information-storage and processing functions to artifacts that are to varying degrees integrated into larger cognitive systems may result in a diminishing of the outsourced function or capacity, as the above example of navigation systems demonstrated. Nicolas Carr (2011) therefore claimed that technology does not make us smart, but makes us stupid. Critics, like Carr, fear that if artifacts do all the cognitive work for us, we will never learn to do it ourselves, or when we have learned to do it ourselves, we might lose that capacity due to a lack of practice. For example, navigation systems decrease the level of detail in our internal cognitive maps, thereby diminishing our capacity to navigate without such devices; constantly using calculators may result in lesser developed calculation skills; and reliable Internet access reduces our internal knowledge base, because when we know information is easily available externally we tend to put less effort into memorizing it.

But in a world where many people have wearable computing devices, one might ask how bad this really is. Of course, there will be moments when we will be decoupled from our devices and then experience that we are less good in remembering facts without access to Google and Wikipedia, performing calculations without a calculator, navigating without Google Maps, and planning without our online diary. However, 
proponents may argue that these are minor drawbacks in relation to what we gain from cognitive technologies. One possible way to look at this situation is by taking a consequentialist view and compare the advantages with the disadvantages. If the advantages outweigh the disadvantages, then the changes to our onboard cognitive capabilities are acceptable.

These worries echo Socrates' critique of written language outlined in Plato's Phaedrus. Socrates argues that written language would erode biological memory as it allows us to store information in the environment, rather than in the brain, in that way making us cognitively lazy. Although Socrates may have a point, ${ }^{4}$ very few people today would think that the development of written language is morally or culturally undesirable, because it has brought us such enormous progress. With written language we may have given up our ability to remember long narratives and our narrative culture (see Rowlands 1999, p 135), but much progress in science, engineering, law, education, and literature would have been impossible without it. If (wearable) computing technologies have similar cognitive and cultural effects, then we might say the advantages outweigh the disadvantages.

An obvious problem with this kind of consequentialist reasoning is that it is difficult to predict what cognitive and cultural consequences (wearable) computing technology will ultimately have. For this reason, much more conceptual and empirical research is needed on this topic (see also Barr et al. 2015). But one likely consequence is that information and computing will become more ubiquitous. I think it is safe to say that the computerization of our lifeworld will continue in the future. When that happens, the cognitive functions of computational artifacts will be integrated deeper into a broader spectrum of our cognitive abilities. Personalized smart devices and smart environments will then complement and transform our onboard cognitive system in more ways, which is already happening with our use of smartphones and tablets. In light of these developments, future conceptual and empirical research should focus on at least the following two questions: (1) what cognitive skills do we want to have in an information society; and (2) how to design these artifacts and systems such that they optimize wellbeing (broadly construed) and reduce possible negative consequences on brain, cognition, and culture?

\subsection{The moral status of cognitive artifacts}

Neuroethicists have argued that interfering with people's brains (either with psychopharmaceuticals, transcranial magnetic stimulation, or deep brain stimulation) might be problematic, as our identity and capabilities are largely determined by the brain. Our brains realize our emotional and cognitive capabilities, which, according to some, are seen as essential for our moral status. ${ }^{5}$ However, we have seen that situated and extended approaches to cognition argue that it is not just our brains that matter for our cognitive capacities. Artifacts and other structures are important, too. So how do cognitive artifacts relate to the notion of moral status? An implication of the extended

\footnotetext{
${ }^{4}$ Somewhat ironically, if Plato did not wrote down Socrates' worries, we would most likely not know about it today.

5 These are by no means the only properties relevant for our moral status. For an overview, see Jaworska and Tannenbaum (2013).
} 
mind thesis is that "in some cases interfering with someone's environment will have the same moral significance as interfering with their person" (Clark and Chalmers 1998, p. 18). For instance, tampering with information in Otto's notebook is the same as tampering with information stored in his brain. Johnny Søraker therefore concludes that "the case with Otto's notebook suggests that information and information technology can have moral status, but only if they are constitutive and irreplaceable in a strong sense" (2007, p. 14). Thus because external information, in some cases, has the same cognitive status as information stored in the brain, it has moral status.

Neil Levy makes a somewhat similar point, but extrapolates further by arguing that:

"Neuroethics focuses ethical thought on the physical substrate subserving cognition, but if we accept that this substrate includes not only brains, but also material culture, and even social structures, we see that neuroethical concern should extend far more widely than has previously been recognized" (2007a, p. 10).

By including material culture into the domain of neuroethics and the cognitive enhancement debate, the scope and ambition of the debate are significantly expanded. I think both Søraker and Levy's suggestions are excellent, but neither conceptualize the further implications of their claims. What does it mean to say that some cognitive artifacts have moral status? One thing it means is that, in addition to traditional legal and moral issues of artifact-ownership, we ought not to interfere with people's distributed minds. This seems particularly the case when people use artifacts to treat their cognitive impairment or decline. Alzheimer's patients, for instance, structure their home-environment such that it compensates their cognitive decline (Dahlback et al. 2013; Drayson and Clark forthcoming). Such patients often have photos of family and friends with indications of their relation to the patient (e.g., daughter), labels on objects and doors, use memory books similar to Otto's notebook, and keep important objects (e.g., checkbooks) always in plain view, not locked away in drawers. Take these people out of (or change) their personalized, self-structured environments and one literally reduces their memory capabilities, which is highly undesirable not just from a healthcare perspective, but also from a moral perspective (see also Drayson and Clark forthcoming).

Generally, the more people depend on external information for their day-to-day cognitive functioning, the deeper it is integrated with their onboard cognitive system. These two aspects, degree of dependency and integration, are proportional to the artifact's moral status. Thus the moral status of cognitive artifacts is, I suggest, a matter of degree and so there is no clear threshold for the moral status of cognitive artifacts. Even artifacts that are shallowly integrated and merely scaffold our thinking are important for the kind of cognitive abilities we have. Levy (2007b, 2011) therefore argues that embedded views equally imply that scaffolding deserves more attention from neuroethicists. He argues that it does not matter whether we call situated cognitive systems embedded/scaffolded or extended/ distributed, what matters is the effect the artifact has on our cognitive capabilities. I agree with Levy, but the effects they have are typically stronger when the dependency is greater and the integration denser. So, I think, it does matter whether cognition is merely embedded or genuinely distributed for the moral status of cognitive artifacts. 
Finally, cognitive scientist Merlin Donald points out that computers "represent a potentially irreversible shift in the cognitive balance of power towards complete external symbol system-based dominance of human cognitive structure" (1991, pp. 355). There indeed seems to be a shift away from storing information internally and towards storing information external to the brain (Sparrow et al. 2011). This means that, in general, if we become more dependent on artifacts for performing our cognitive tasks, their moral status increases. In section 4.1, I outlined that an unintended consequence of artifact-use might be diminished onboard cognitive capabilities in some domains, which, in turn, results in a higher dependency on artifacts. If this trend continues, which Donald (1991) and Sparrow et al. (2011) seem to suggest, we may infer that in the future, artifacts will become more important for our day-to-day cognitive functioning and thus obtain a higher moral status.

\subsection{Personal identity}

Offloading information-storage functions to external media is a typical human feature that shapes not only our cognitive system but also our personal identity in profound ways. Clark and Chalmers recognized that "the information in Otto's notebook, for example, is a central part of his identity as a cognitive agent" (1998, p. 18). Personal identity is a complex concept and I cannot do it justice in this short section. But whatever psychological view one takes on personal identity, it is clear that memory plays a central role. Memory is important to both the diachronic and synchronic problem in the debate on personal identity. The diachronic problem concerns the psychological continuity of one's personal identity over time, whereas the synchronic problem concerns the psychological features that characterize a person at one time.

In relation to the diachronic problem, some theorists focus on narrative memory, arguing that who we are, i.e., our personal identities, can be seen as the narrative construction of our past personal experiences, our autobiographical memories. On this neo-Lockean view, our narrative is seen as constitutive of our personal identity. Given this focus on memory, there is an obvious link to extended mind theory, which has widely conceptualized extended memory (Wilson and Lenart 2014). Autobiographical memories are not always located in the brain, but sometimes materialized into artifacts such as photos, videos, and other significant objects like books, furniture, souvenirs, or artworks (Van den Hoven 2014). An interesting example here is SenseCam, a small wearable camera typically worn around one's neck that automatically takes a picture with a certain frequency, creating a visual narrative of one's day-to-day life. SenseCams are used by "normal" people and by those with cognitive impairments. Some patients with amnesia or Alzheimer's disease use SenseCam as a substitute for their biological memory. For such patients, the visual narrative created by the device is literally part of their life-narrative and thus constitutive of their personal identity. ${ }^{6}$

However, it is not just autobiographical memories that are important for personal identity. One's memory capabilities in the here-and-now are also relevant. Therefore, in relation to the synchronic problem, we should take into account the memory aids that are not directly related to autobiographical memory. The above example of Alzheimer's patients structuring their home-environment such that it compensates their cognitive

\footnotetext{
${ }^{6}$ But see Lynne Rudder Baker (2009) for a critical discussion of extended personhood.
} 
decline is a case in point. Their use of photos, labels, memory books, and deliberately placing objects at easily perceivable locations can be seen as cognitively "constitutive and irreplaceable in a strong sense" (Søraker 2007, p. 14). Take these people out of (or change) their self-structured environments and one reduces not just their memory but also their personal identity. This does not only happen with patients, but also with cognitively healthy people. Most people store a lot of information in their smartphones or tablets (e.g., music, documents, pictures, videos, etc.) and use it for navigating, planning their daily tasks, calculating, searching for information, and many other things. These functions are not so much concerned with autobiographical memory, but more with working memory in day-to-day tasks. Most people literally would not be able to do some of these tasks without the aid of their personalized computing device. So our abilities and personal identity are relational (Mackenzie and Walker 2015) and are thus partly defined by the cognitive technologies we use.

\section{Conclusion}

Humans enhance their cognitive abilities not just with psychopharmaceuticals but also with external artifacts. These have important moral aspects that have been neglected by both the cognitive enhancement debate and situated cognition theory. This paper developed a first attempt to fill this gap by conceptualizing and evaluating some of the consequences of cognitive artifacts on brain, cognition, and culture; the moral status of cognitive artifacts; and the effects they have on personal identity. These are just three possible topics for moral reflection and further developing them and others would strengthen the rather thin ties between and enrich both the cognitive enhancement debate and situated cognition theory. The take home message is therefore twofold: (a) neuroethics needs to pay more attention to cognitive artifacts, and (b) to fully conceptualize situated cognitive systems theorists in this field need to take into account their moral aspects. Thus in future research, situated cognition theory may provide rich case studies and the neuroethics of cognitive enhancement some of the conceptual tools to analyze these case studies.

Acknowledgments I would like to thank the Collective Cognition Team, particularly John Sutton, at the Centre for Cognition and its Disorders at Macquarie University for funding to write this paper. I also wish to thank Sadjad Soltanzadeh, Regina Fabry, and two anonymous reviewers for helpful feedback on an earlier version of this paper. This paper was presented at the 2015 Australasian Association of Philosophy conference held in Sydney. I like to thank the audience for their questions and feedback.

\section{References}

Baker, L. R. (2009). Persons and the extended mind thesis. Zygon, 44(3), 642-658.

Barr, N., Pennycook, G., Stolz, J. A., \& Fugelsang, J. A. (2015). The brain in your pocket: evidence that smartphones are used to supplant thinking. Computers in Human Behavior, 48, 473-480.

Bostrom, N., \& Sandberg, A. (2009). Cognitive enhancement: methods, ethics, regulatory challenges. Science and Engineering Ethics, 15(3), 311-341.

British Medical Association. (2007). Boosting your brainpower: Ethical aspects of cognitive enhancement. London: BMA. 
Buckner, C. (2015). A property cluster theory of cognition. Philosophical Psychology, 28(3), 307-336.

Burnett, G. E., \& Lee, K. (2005). The effect of vehicle navigation systems on the formation of cognitive maps. In G. Underwood (Ed.), Traffic and transport psychology: Theory and application (pp. 407-418). Amsterdam: Elsevier.

Carr, N. (2011). The shallows: What the internet is doing to our brains. New York: W.W. Norton \& Company. Clark, A. (2003). Natural-born cyborgs: Minds, technologies, and the future of human intelligence. Oxford: Oxford University Press.

Clark, A. (2007). Re-inventing ourselves: the plasticity of embodiment, sensing, and mind. Journal of Philosophy and Medicine, 32(3), 263-282.

Clark, A., \& Chalmers, D. (1998). The extended mind. Analysis, 58(1), 7-19.

Clausen, J., \& Levy, N. (Eds.). (2015). Handbook of neuroethics. Dordrecht: Springer.

Dahlback, N., Kristiansson, M., \& Stjernberg, F. (2013). Distributed remembering through active structuring of activities and environments. Review of Philosophy and Psychology, 4(1), 153-165.

Donald, M. (1991). Origins of the modern mind: Three stages in the evolution of our cognitive system. Cambridge: Harvard University Press.

Drayson, Z., \& Clark, A. (forthcoming). Augmentation, agency, and the spreading of mental state. Consciousness and Cognition.

Farah, M. J., Illes, J., Cook-Deegan, R., Gardner, H., Kandel, E., King, P., Parens, E., Sahakian, B., \& Wolpe, P. R. (2004). Neurocognitive enhancement: what can we do and what should we do? Nature Reviews Neuroscience, 5(5), 421-425.

Hansson, S. O. (2005). Implant ethics. Journal of Medical Ethics, 31(9), 519-525.

Harris, C. B, Keil, P. G., Sutton, J., \& Barnier, A. J. (2010). Collaborative remembering: When can remembering with others be beneficial? In W. Christensen, E. Schier \& J. Sutton (Eds.), Proceedings of the 9th conference of the Australasian society for cognitive science (pp. 131-134).

Haugeland, J. (1998). Mind embodied and embedded. In J. Haugeland (Ed.), Having thought: Essays in the metaphysics of mind (pp. 207-240). Cambridge: Harvard University Press.

Heersmink, R. (2014). The metaphysics of cognitive artefacts. Philosophical Explorations. doi:10.1080/ 13869795.2014.910310.

Heersmink, R. (2015). Dimensions of integration in embedded and extended cognitive systems. Phenomenology and the Cognitive Sciences, 14(3), 577-598.

Hildt, E., \& Franke, A. G. (Eds.). (2013). Cognitive enhancement: An interdisciplinary perspective. Dordrecht: Springer.

Hutchins, E. (1995). Cognition in the wild. Cambridge: MIT Press.

Ihde, D. (1990). Technology and the lifeworld: From garden to earth. Bloomington: Indiana University Press.

Illes, J., \& Sahakian, B. J. (Eds.). (2011). Oxford handbook of neuroethics. Oxford: Oxford University Press.

Jaworska, A., \& Tannenbaum, J. (2013). The grounds of moral status. In E. N. Zalta (Ed.), The Stanford encyclopedia of philosophy (Summer 2013 Edition). URL: http://plato.stanford.edu/archives/sum2013/ entries/grounds-moral-status/.

Kirsh, D., \& Maglio, P. (1994). On distinguishing epistemic from pragmatic actions. Cognitive Science, 18(4), 513-549.

Kiverstein, J., \& Clark, A. (2009). Introduction: mind embodied, embedded, enacted: one church or many? Topoi, 28(1), 1-7.

Latour, B. (1994). On technical mediation: philosophy, sociology, genealogy. Common Knowledge, 3(2), 2964.

Levy, N. (2007a). Rethinking neuroethics in the light of the extended mind thesis. American Journal of Bioethics, 7(9), 3-11.

Levy, N. (2007b). Neuroethics: Challenges for the 21th century. Cambridge: Cambridge University Press.

Levy, N. (2011). Neuroethics and the extended mind. In J. Illes \& B. J. Sahakian (Eds.), Oxford handbook of neuroethics (pp. 285-294). Oxford: Oxford University Press.

Lynch, G., Palmer, L. C., \& Gall, C. M. (2011). The likelihood of cognitive enhancement. Pharmacology Biochemistry and Behavior, 99(2), 116-129.

Mackenzie, C., \& Walker, M. (2015). Neurotechnologies, personal identity, and the ethics of authenticity. In J. Clausen \& N. Levy (Eds.), Handbook of neuroethics (pp. 373-392). Dordrecht: Springer.

Meacham, D. (2015). The subject of enhancement: augmented capacities, extended cognition and delicate ecologies of the mind. The New Bioethics, 21(1), 5-19.

Menary, R. (2010a). Introduction to the special issue on 4E cognition. Phenomenology and the Cognitive Sciences, 9(4), 459-463.

Menary, R. (2010b). Dimensions of mind. Phenomenology and the Cognitive Sciences, 9(4), 561-578. 
Metzinger, T., \& Hildt, E. (2011). Cognitive enhancement. In J. Illes \& B. J. Sahakian (Eds.), Oxford handbook of neuroethics (pp. 245-264). Oxford: Oxford University Press.

Norman, D. (1991). Cognitive artifacts. In J. M. Carroll (Ed.), Designing interaction: Psychology at the human-computer interface (pp. 17-38). Cambridge: Cambridge University Press.

Norman, D. (1993). Things that make us smart: Defending human attributes in the age of the machine. New York: Basic Books.

Pitt, J. C. (2014). Guns don't kill, people kill: Values in and/or around technologies. In P. Kroes \& P. P. Verbeek (Eds.), The moral status of technical artefacts (pp. 89-101). Dordrecht: Springer.

Rowlands, M. (1999). The body in mind: Understanding cognitive processes. Cambridge: Cambridge University Press.

Rowlands, M. (2010). The new science of the mind: From extended mind to embodied phenomenology. Cambridge: MIT Press.

Rupert, R. (2004). Challenges to the hypothesis of extended cognition. Journal of Philosophy, 101(8), 389428.

Santoni de Sio, F., Faulmüller, N., \& Vincent, N. (2014). How cognitive enhancement can change our duties. Frontiers in Systems Neuroscience. doi:10.3389/fnsys.2014.00131.

Savulescu, J., \& Bostrom, N. (Eds.). (2009). Enhancement of human beings. Oxford: Oxford University Press.

Søraker, J. H. (2007). The moral status of information and information technology: A relational theory of moral status. In S. Hongladarom \& C. Ess (Eds.), Information technology ethics: Cultural perspectives (pp. 1-19). Hershey: Idea Group Publishing.

Sparrow, B., Liu, J., \& Wegner, D. M. (2011). Google effects on memory: Cognitive consequences of having information at our fingertips. Science, 333(6043), 776-778.

Sterelny, K. (2010). Minds: Extended or scaffolded? Phenomenology and the Cognitive Sciences, 9(4), 465481.

Sternberg, R. J., \& Preiss, D. D. (Eds.). (2005). Intelligence and technology: The impact of tools on the nature and development of human abilities. New Jersey: Erlbaum.

Sutton, J. (2006). Distributed cognition: Domains and dimensions. Pragmatics and Cognition, 14(2), 235247.

Sutton, J. (2010). Exograms and interdisciplinarity: History, the extended mind, and the civilizing process. In R. Menary (Ed.), The extended mind (pp. 189-225). Cambridge: MIT Press.

Sutton, J., Harris, C. B., Keil, P., \& Barnier, A. J. (2010). The psychology of memory, extended cognition, and socially distributed remembering. Phenomenology and the Cognitive Sciences, 9(4), 521-560.

Turner, D. C., \& Sahakian, B. J. (2006). Neuroethics of cognitive enhancements. BioSocieties, 1(1), 113-123.

Van den Hoven, E. (2014). A future-proof past: designing for remembering experiences. Memory Studies, $7(3), 370-384$.

Ward, D., \& Stapleton, M. (2012). Es are good: Cognition as enacted, embodied, embedded, affective, and extended. In F. Paglieri (Ed.), Consciousness in Interaction: The role of the natural and social context in shaping consciousness (pp. 89-104). Amsterdam: John Benjamins.

Wegner, D. M. (1995). A computer network model of human transactive memory. Social Cognition, 13(3), 319-339.

Wheeler, M. (2011). Thinking beyond the brain: educating and building, from the standpoint of extended cognition. Computational Cultures, 1.

Wilson, R. A., \& Lenart, B. A. (2014). Extended mind and identity. In J. Clausen \& N. Levy (Eds.), Handbook of neuroethics (pp. 423-439). Dordrecht: Springer. 